\title{
Global Access to Environmental Information
}

\author{
Ralf Kramer, Tom Quellenberg \\ Forschungszentrum Informatik (FZI) \\ Haid-und-Neu-Str. 10-14, D-76131 Karlsruhe, Germany. Telephone: \\ (+49) 721 9654-722. Fax: -709. email: kramer/quelle@fzi.de
}

\begin{abstract}
Environmental applications deal with huge numbers of knowledge domains. In order to cope with the increasing number of highly diverse data and information sources, explicit modelling of meta-data is mandatory. This paper focuses on how data in an existing meta-data model, originally developed for German authorities, can be accessed in widearea networks. The current implementation combines navigational access methods via Mosaic and declarative access through SQL. The system architecture and query processing in that architecture are described. Lessons learned from the initial system concern the necessity for design recovery when using an existing meta-data model and experiences obtained from using the stateless World-Wide Web (WWW) environment in database applications. Looking ahead, we sketch the active information system that will support access to semantically heterogeneous information sources in the future.
\end{abstract}

\section{Keywords}

Database systems, environmental data catalogue, meta-data, world-wide web

\section{INTRODUCTION}

All over the world, there is an increasing awareness for the importance of our natural environment. Environmental protection shows up in environmental politics, which is supported by environmental information systems run by state authorities. Environmental questions touch upon a large number of fairly different knowledge domains. Hence, such systems have to deal with geographic information, measurement data from different application areas and other kinds of data. Due to the decentralized organisation of the different German states, it comes as no surprise, that the authorities are organized in different ways and have developed different terminologies. In order to cope with these diverging organizations and terminologies, an environmental data catalogue (UDK) has been developed to account for the global significance of environmental affairs. This meta information system is in the process of being standardized for all German states and in addition, Austria. Current implementations of the UDK basically deliver information on the data stored in 
other systems, or information on whom to call (e.g., via phone). In general, they do not yet support any direct access to the original data.

Besides this application pull, there is a technological push that will determine the potential for future applications of such meta information systems. Among the most important ones are global networks. Along with these global networks come user friendly public domain tools like Mosaic for the World-Wide Web, that provide convenient access to all kinds of information using a graphical user interface.

In this paper, we describe how these two approaches, namely the meta information system UDK and graphical user interfaces for the WWW, have been combined within the project GLOBUS/UDK* [Kramer 1994]. The remainder of this paper is organized as follows: In the next section we describe these two cornerstones in more detail. In Section 3, the specific requirements for GLOBUS/UDK are given, the system's current architecture is presented in some more detail, and several examples illustrate the querying of meta-data. Section 4 summarizes the lessons learned so far from combining an already existing meta information model and system with a new interface that supports access from WWW. In Section 5, we compare our approach with related work. Section 6 presents the final conclusions and an outlook on future work.

\section{CORNERSTONES}

\subsection{Environmental Data Catalogue (UDK)}

The information required to answer many environmental questions (e.g., environmental protection) is often found in different knowledge domains located on different systems. Thus, in order to find the data and the system to answer a certain question, we first have to find out which information is accessible and where and how to find it. That is, we need some meta-data. Hence, a meta information system is required that consists of two main parts, namely the meta-data itself and an application program that accesses the meta-data.

The environmental data catalogue UDK and the corresponding UDK application program is a meta information system that serves three main purposes [Schütz 1994]: It should include information on all data that are relevant for environmental systems in order to be able to deliver complete answers; it should describe the quality of the original data and it should support the access to the underlying original data.

The UDK application program currently used (e.g., at the Landesanstalt für Umweltschutz (LfU), Karlsruhe, and other governmental agencies) stores its data in a relational database system. At the user front-end, the user first chooses either a catalogue that reflects the organization of the authorities in his/her specific state or country, or a catalogue that reflects certain subject-specific organizations, e.g., all data concerning air, soil or water (we will come back to these access paths in Section 3.3). Then, (s)he can reach into the meta-data directly or via additional search terms that are attached to the meta-data via something like a thesaurus. As a result of such queries, the meta-data found are presented. Since, very little of the underlying original data is accessible online today, addresses and telephone numbers are the prime means to obtain the desired information 'offline'.

*GLOBUS/UDK was funded by Umweltministerium Baden-Württemberg. 


\subsection{World-Wide Web and its Tools}

The World-Wide Web (WWW) [Berners-Lee 1993b] is a hypertext- and multimediasystem for documents including text, pictures, movies or audioclips. Information resides on servers that are accessible, e.g., via the Internet using suitable viewers (e.g., Mosaic [NCSA 1994], NetScape). The only structuring mechanism for sets of documents is the facility to place links (Universal Resource Locators or URL) in documents leading to other documents. They consist of a protocol specification, the server address and the location of the document at the server. By following such a link, the information referenced is transfered from the server to the client and interpreted by the viewer.

Most documents on the WWW are written as HTML documents using the hypertext markup language (HTML) [Berners-Lee 1993a]. However, many client programs are able to understand further formats (e.g., plain text, postscript). The HTML format allows a structural description of text elements (headings, sections), text styles (bold, italic), and the inclusion of pictures within the text. Links to other documents can connect to text or pictures. An extension of this format (HTML+) allows the description of forms, with fields for editing text and buttons. The WWW client displays this form, the user fills it with information and then this information is sent back to the server. At the server, a specified program for this form is called via the Common Gateway Interface (CGI) [McCool 1994], which interprets the information. The output of this program is made available by the server to clients as a new HTML document. Thereby, hypertext pages can be generated dynamically, which allows a very flexible generation and presentation of information. The programs started via CGI can be written in any language available at the server (e.g., shell-scripts, perl, C, C++, ...).

With the great success and the huge number of WWW servers all around the world, some limitations and problems of the current system become apparent. One problem is the lack of access control to information available on a server: Information offered via a WWW server can be read by everyone on the net. It is not yet possible to limit page access to a specified group of people. Another problem is the lack of any structuring elements for the hypertext net; there is only a flat net of pages and links. Furthermore, these links are unidirectional only. A link cannot be updated if the page it refers to is changed or moved to another location.

\section{USING WWW TO ACCESS ENVIRONMENTAL META-DATA}

\subsection{Requirements}

The requirements for GLOBUS/UDK can be summarized as follows:

1. The modelling of UDK meta-data remains unchanged.

2. We assume that UDK meta-data is centralized both logically and physically.

3. Access across the wide-area network is read-only.

4. Adding new UDK meta-data and updating existing one is left to the UDK application program that runs on a PC. This program can be used for local read-accesses as well.

5. The functionality across the net is similar to the one of the existing UDK program.

6. The system architecture has to be based on WWW communication and tools. 


\subsection{System Architecture}

Given the cornerstones and requirements as presented in Sections 2 and 3.1, an initial system had to be designed and implemented that allows access to UDK data stored in a relational database system across a wide-area network. This data should be accessible using Mosaic clients and the other WWW techniques described in Section 2.2.

For storing meta-data that is accessible via Mosaic, there are two alternatives. Either, data can be extracted from the database system periodically to build static HTML pages, or the database can be queried on a user's request and subsequently, the HTML pages are built dynamically.

The main problem with the first alternative is that Mosaic only supports navigational access and a full text search on the current page, which obviously is not sufficient. From the user's perspective, the second alternative offers several advantages such as declarative querying and access to the most up-to-date data. Thus, the second alternative is chosen.

Figure 1 gives a more detailed outline of the current system. The WWW client (i.e., Mosaic) accesses the WWW server across the network, which, unfortunately is not yet the Internet; hence, we cannot provide the URL here. The WWW server provides a single static HTML page named start_udk as the entry point. For all subsequent UDK accesses, the WWW server uses the CGI as described in Section 2.2 and a database precompiler program written in C. Approaches like GSQL [Ng 1993] do not yet provide the means to implement state-of-the-art user interfaces (see section 5.2). Thus, we rely on the precompiler approach, although such a solution is difficult to debug and to maintain. This program named $u d k_{-}$access retrieves the relevant data from the relational database system (Oracle Version 7) and builds the resulting HTML pages dynamically. These pages are presented to the user via Mosaic.

\subsection{Querying Meta-data}

In this section, we present examples for simple and complex queries that access the metadata. To facilitate readers that are unfamiliar with German, the description of the search forms are restricted to those found in figure 2, which are easier to understand. First, let's have a look at how the meta-data is organized in the UDK.

All UDK meta-data is organized hierachically. On the first level, different countries like Germany and Austria are distinguished, on the second one, the user chooses a state within a country, e.g., Baden-Württemberg. Finally, for each state, there are two catalogues to choose from: the first one reflects each state's organization of authorities, the second one organizes the meta-data according to their content.

In the middle part of the left screendump shown in figure 2, we see the user's initial choice among two catalogues named $U D K$ and $G D K$ for the states Baden-Württemberg $(B W)$ and Niedersachsen $(N I)$ located in Germany $(G E R)$. Having made the initial choice among the catalogues available, the user may search for addresses or so-called $U D K$ objects. Simple searches can be used to locate addresses or UDK-objects, while more complex searches can only be used with UDK-objects. An example of a complex search is found in figure 2 .

For simple, straight-forward queries, the search parameters, which may include the usual SQL wildcards, are mapped to several attributes of different relations within the precompiler program udk_access. This hides the complexity of the underlying data model from 


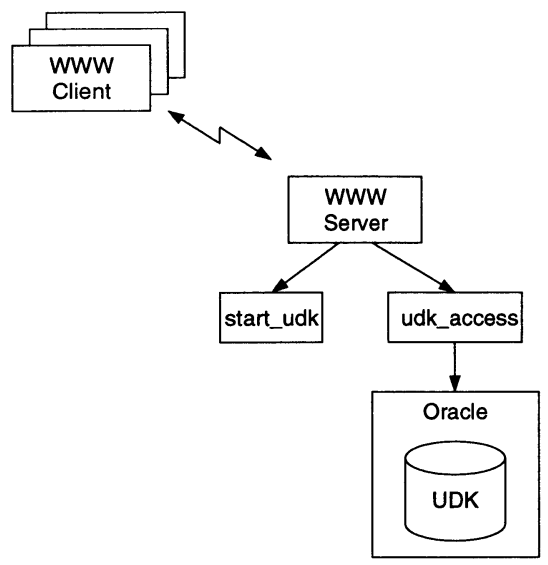

Figure 1 A detailed Look of the current System

the user. The query result is presented in a different form that is generated dynamically depending on the result tuples.

The right part of figure 2 depicts an example of a more complex search. Basically, each of the 7 search attributes (i.e., Objektname, Adresskennzeichen, ...) target several attributes of a single relation. In combination with the search parameters, the user can choose among a set of comparison operators (e.g., $=, \leq,<, \ldots$ ). The triples search attribute, comparison operator, search parameter are combined using intersection and union operations (i.e., $A N D$ and $O R$ ). Obviously, this flexible and powerful mechanism requires dynamic SQL within the program $u d k_{-} a c c e s s$ and hence, a more elaborate implementation than the simple searches sketched above. However, the underlying complexity remains completely hidden from the user.

As a result of both simple and complex queries, the user first receives a list that includes important information relating to addresses and UDK-objects. In these lists, the user can choose those addresses and objects from which (s)he wants to obtain further details. Then, as the second result, all relevant attributes from the underlying relations are presented in another form that has been generated dynamically.

\section{LESSONS LEARNED}

In this section, we discuss the most important lessons obtained from the initial system described in Section 3. The lessons include the impact of poor database design on the implementation effort, deficiencies of the current WWW tools for implementing stateof-the-art database user interfaces, and the impact of using a stateless communication paradigm.

In contrast to many other environmental (meta-) information systems we started with an already existing meta-data model. The starting point of our project were some 15 base relations that comprise the UDK's most important data. Unfortunately, only a non- 


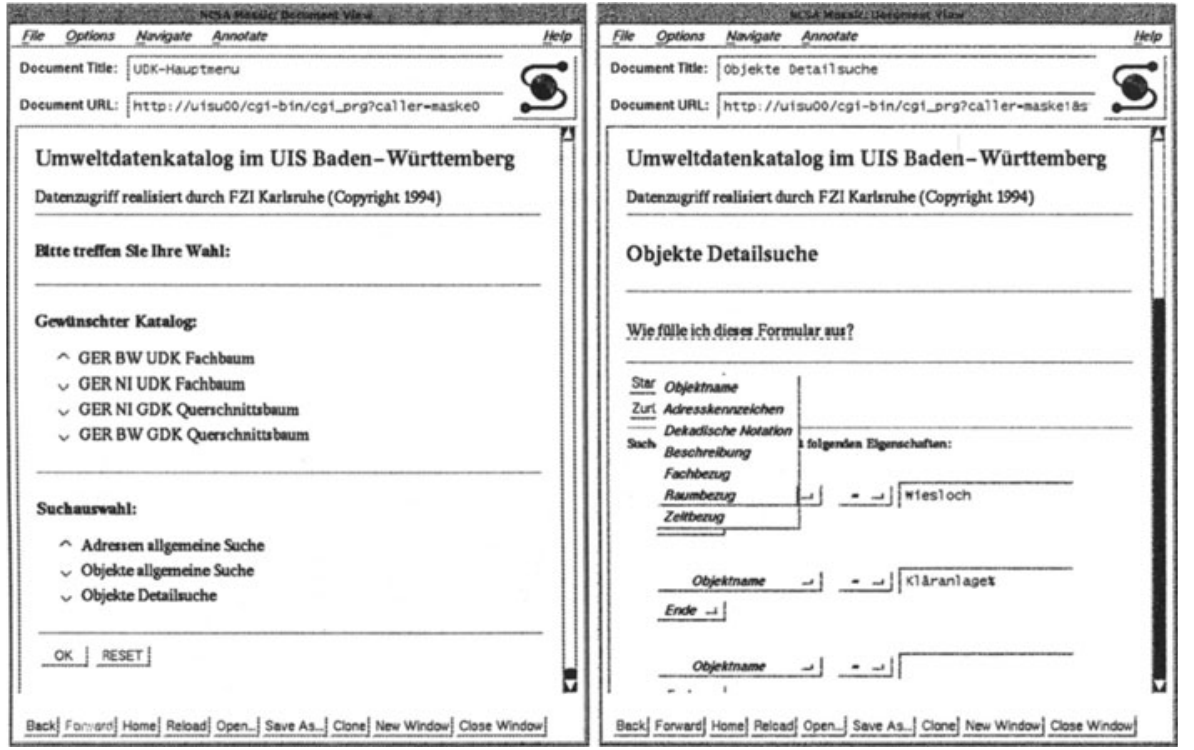

Figure 2 Choosing among different Data Catalogues and Complex UDKObject Search

standard graphical diagram and the tables (for the relational database system Oracle, Version 7) were available. Some pecularities of the database design (e.g., lack of data independency) were hard to understand by simply examining the schema, i.e., without looking at the data. Thus, we used the UDK's PC implementation to run against the same meta-data as in our implementation in order to find the "correct" SQL queries. That is, those queries that yield the same results in our application as in the PC implementation. In our case, the existing meta-data model required a design recovery [Chikofski 1990] (usually refered to as the first step of reverse engineering process) in order to implement the new WWW/Mosaic application.

As already pointed out at the end of Section 3.3, current WWW tools require a precompiler to implement state-of-the-art user interfaces. That is, interfaces in which the format of query results do not match the search format. With the precompiler approach, even minor changes in the database design or in the generated HTML pages require a substantial knowledge of the implementation. Thus, a higher abstraction level and some sort of interpretation or precompilation to the lower level are mandatory to achieve a better maintainability.

Different from traditional database applications (stand-alone and client-server applications), communication in WWW/Mosaic environments is stateless. Thus, there is no notion of a session or (ACID-) transaction across the net, i.e., when a WWW client (like Mosaic) demands a HTML document from a WWW server. Classical database transactions only take place locally at the server's site within the precompiler application (see figure 1). We agree with the conclusions of [Eichmann 1994] that - as a side-effect - user 
interfaces are likely to become more simplistic. For example, the move to a stateless environment limits the number of actions a window or page is able to process. Approaches like the one taken in Zelig [Varela 1994] to generate database applications dynamically are another promising approach to allow for adaptable user interfaces across the net.

\section{RELATED WORK}

In this section, we place our work in the context of other environmental information systems (Section 5.1) and - independent from a specific application area - in the context of database access in wide-area networks (Section 5.2). The list of approaches, concepts, and systems discussed does not claim to encompass all relevant work in the area. We restrict the discussion to those that we think to bear the most direct relationships to our problem and solution.

\subsection{Environmental Information Systems}

In this section, we discuss those environmental information systems that support - or, to be more precise, at least intend to support - the access to environmental data in wide-area networks. In contrast to most other approaches, we employ an already existing and fairly elaborate meta-data model.

Up to now, only one approach to make the UDK available across the net has been published [Lessing 1994]. [Lessing 1994] mainly discusses issues that are complimentary to our focus, namely security and authorization problems when accessing the UDK and the underlying original data. In contrast, we concentrated on making the UDK's metadata available using WWW tools. Hence, we have not yet addressed the security issues mentioned in section 2.2. We feel it worthwhile to examine more closely how to incorporate the approach [Lessing 1994] in our system.

The CERES Global Knowledge Network (CERES-GKN) [CERC 1994] aims at supporting the environmental-conscious design of products [Evans 1994]. The intended user communities are - among others - companies and their engineers, and not authorities as in our implementation. While the users are different, the first implementation of CERESGKN is likely to be based on WWW tools as well. So, although the intended audiences are different, the techniques used or intended to be used, are similar with ours.

The Consortium for International Earth Science Information Network (CIESIN) aims easing the access to information and data, used in facilitating the understanding of human interactions with the environment [Zimmermann 1994]. The intended audience for this application are mainly scientists and the public. As mentioned previously, CERES-GKN focuses on decision making in business and industry and GLOBUS/UDK - at least in the short run - on providing information for authorities. CIESIN offers both access to data and meta-data.

According to [Crossley 1994], the Australian Environmental Resources Information Network ERIN [ERIN 1994] uses Wide Area Informations Server WAIS to access environmental data. WAIS server use indexes for each word in every document; the index is used for query processing. In [Crossley 1994], the importance of meta-data and advantages of using database systems to manage the meta-data are discussed. With ERIN's focus on spatial queries, a relational database system is used to store both data and meta-data 
[Boston 1994]. The system architecture is very similar to the one we use (see figure 1). Just like in our approach (see section 3.3), ERIN queries first deliver a summary report before the user can select further information on individual records [Boston 1994, Section 2].

\subsection{Database Access in Wide-Area Networks}

For database access via WWW-servers, systems like GSQL [Ng 1993] support the generation of forms and queries. Unfortunately, they offer only a basic functionality for the presentation of the results. In particular, it is impossible to use results of queries in detailed query forms or to combine navigational and declarative access flexibly. To summarize, although such tools provide a higher abstraction level, they do not meet the requirements of today's user-interfaces like the one sketched in section 3.3 and shown in figure 2 .

In the Repository Based Software Engineering (RBSE) project, a repository based on meta-data is accessible via Mosaic and the WWW. Similar to our system, the current MORE system [Eichmann 1994] is a reimplementation of a 'conventional' database application. Thus, the lessons learned are similar to ours, with the notable exception that we did not reengineer the database design.

From a database system point of view, several architectural approaches such as, distributed databases, multi-databases, and federated databases, have been proposed to solve the problem of heterogeneity at technical, syntactical, and semantical levels. We refer to [Litwin 1990, Sheth 1990] for an overview. With its present lack of any active capabilities, our approach can be characterized as a federation, i.e., a number of loosely coupled systems that retain their autonomy while participating in the federation. Unfortunately, federated systems are unable to solve the problem of semantic heterogeneity [Papazoglou 1992]. Thus, a certain degree of activity will be required to achieve the desired interoperability.

\section{CONCLUSIONS AND FUTURE WORK}

In this paper, we have presented the approach taken in GLOBUS/UDK to access environmental meta-data in wide area networks. Demonstrations and first user tests of our system showed that we have implemented a viable approach combining navigational and declarative accesses using technology that is currently available. The meta-data is stored in a relational database system. The application itself, allows for a combination of navigational and declarative access by combining the capabilities of Mosaic and SQL.

The vision or goal of the final system can be described as an active information system that supports access to semantically heterogeneous information sources in multiple user-specific terminologies. The current UDK serves as the core and starting point for a - logically - centralized general meta-data repository. Currently, so-called intelligent and cooperating information systems [Papazoglou 1992] are viewed as the most promising approach to cope with the problems of system federations. We plan to address the severe problem of semantic heterogeneity and semantic interoperability [Jamil 1994] in the future.

Acknowledgements: The students K. Ateyeh, O. Scheffczyk, and M. Walz were instrumental in implementing GLOBUS/UDK. We gratefully acknowledge their support. Special thanks are due to the other members of FZI's and the University of Karlsruhe's Infor- 
mation Systems Group joint working group on system federations (Dr. G. von Bültzingsloewen, A. Koschel, C. Reck, B. König-Ries, R. Schmidt, D. Theobald, and Dr. M. Wallrath) for the stimulating discussions. Their suggestions and Prof. Dr. P. C. Lockemann's comments on an earlier version of this paper significantly improved its focus.

\section{REFERENCES}

[Berners-Lee 1993a] T. Berners-Lee. Hypertext mark-up language. Technical report, CERN, http://info.cern.ch/hypertext/WWW/MarUp/MarkUp.html, 1993.

[Berners-Lee 1993b] T. Berners-Lee. The world wide web initiative. Technical report, CERN, http://info.cern.ch/hypertext/WWW/Theproject.html, 1993.

[Boston 1994] Tony Boston and David Stockwell. Interactive species distribution reporting, mapping and modelling using the World Wide Web. In Second International World Wide Web (WWW) Conference '94: Mosaic and the Web, Chicago, USA, October 1994. http://kaos.erin.gov.au/database/WWW-Fall94/species_paper.html.

[Chikofski 1990] E.J. Chikofski and J.H. Cross II. Reverse Engineering and Design Recovery: A Taxonomy. IEEE Software, 7(1):13-18, January 1990.

[CERC 1994] The Concurrent Engineering Research Center, West Virginia University, USA; The CERTIA Research Center, University of Roma at Torvergata, Italy; The Advanced Research Laboratory, Pennsylvania State University, USA. Inaugural Workshop for the CERES Global Knowledge Network, Berkley Springs, West Virginia, USA, April 1994. http://www.cerc.wvu.edu/ceres/inaugural_workshop_index.html.

[Crossley 1994] David Crossley. WAIS throug the Web Discovering Environmental Information. In Second International World Wide Web (WWW) Conference '94: Mosaic and the Web, Chicago, USA, October 1994. http://www.ncsa.uiuc.edu/SDG/IT94/Proceedings/Searching/crossley/paper.html.

[Eichmann 1994] D. Eichmann, T. McGregor, and D. Danley. Integrating Structured Databases Into the Web: The MORE System. In First International Conference on the World-Wide Web, Geneva, Switzerland, May 1994. CERN. Preliminary Proceedings, http://www1.cern.ch/WWW94/PrelimProcs.html.

[Elmagarmid 1990] Ahmed K. Elmagarmid and Calton Pu. Special issue on heterogeneous databases. ACM Computing Surveys, 22(3), September 1990.

[Evans 1994] S. Evans and E.P. Rose. Environmentally Conscious Design Using a Global Knowledge Network. Technical report, Cranfield University, CIM Institute, UK, 1994.

[ERIN 1994] ERIN General Information. http://kaos.erin.gov.au/general/general.html.

[Jamil 1994] Hasan Jamil and Paul Johannesson. Sematic interoperability - context, issues and research directions. In Michael Brodie, Mathias Jarke, and Michael Papazoglou, editors, Proc. of the 2nd Intl. Conference on Cooperative Information Systems, pages 180-191, Toronto, Canada, May 1994.

[Kramer 1994] R. Kramer and T. Quellenberg. Global Access to Environmental Information. FZI Report 9/94, Forschungszentrum Informatik (FZI), Karlsruhe, Germany, December 1994.

[Litwin 1990] W. Litwin, L. Mark, and N. Roussopoulos. Interoperability of multiple autonomous databases. In ACM Computing Surveys [Elmagarmid 1990], pages 267-293.

[Lessing 1994] H. Lessing and T. Schütz. Der Umwelt-Datenkatalog als Instrument zur Steuerung von Informationsflüssen. In L.M. Hilty, A. Jaeschke, B. Page, and A. Schw- 
abl, editors, Informatik für den Umweltschutz; 8. Symposium, Hamburg 1994; Band I, Umwelt-Informatik Aktuell, pages 159-167, Marburg, 1994. Metropolis.

[McCool 1994] Rob McCool. Common gateway interface overview. Technical report, National Center for Supercomputiong Applications (NCSA), http://hoohoo.ncsa.uiuc.edu/cgi/overview.html, 1994.

[NCSA 1994] NCSA. Introduction to NCSA Mosaic for X. Technical report, National Center for Supercomputing Applications (NCSA), http://www.ncsa.uiuc.edu/SDG/Software/Mosaic/Docs/d2-intro.html, 1994.

[Ng 1993] Jason Ng. GSQL: A Mosaic-SQL gateway. Technical report, National Center for Supercomputing Applications (NCSA), http://www.ncsa.uiuc.edu:80/SDG/People/jason/pub/gsql/, 1993.

[Papazoglou 1992] Mike P. Papazoglou, Steven C. Laufmann, and Timos K. Sellis. An organizational framework for cooperating intelligent information systems. Intl. Journal on Intelligent and Cooperative Information Systems, 1(1):169-202, March 1992.

[Schütz 1994] T. Schütz and R. Böhm. Die Datenstrukturierung des Metainformationssystems Umwelt-Datenkatalog. In H. Kremers, editor, Umweltdatenbanken, number 5 in Praxis der Umweltinformatik, pages 245-258, Marburg, 1994. Metropolis.

[Sheth 1990] Amit P. Sheth and James A. Larson. Federated database systems for managing distributed, heterogenous, and autonomous databases. In ACM Computing Surveys [Elmagarmid 1990], pages 183-236.

[Varela 1994] C.A Varela and C.C. Hayes. Zelig: Schema-Based Generation of Soft WWW Database Applications. In First International Conference on the World-Wide Web, Geneva, Switzerland, May 1994. CERN. Preliminary Proceedings, http://www1.cern.ch/WWW94/PrelimProcs.html.

[Zimmermann 1994] F. Zimmermann and P. Young. Information for a Changing World: CIESIN's Use of WWW to Facilitate Access to Data and Information Relevant to Human Interactions in The Environment. In Second International WWW Conference '94, Chicago, USA, October 1994. http://www.ncsa.uiuc.edu/SDG/IT94/Proceedings/EarSci/zimmerman/\% -zimmerman.html.

\section{BIOGRAPHY}

Ralf Kramer is project manager and Executive Vice-Director of the database research group at FZI, Karlsruhe. He holds a Diploma degree (Dipl.-Wi.-Ing.) and a Ph.D. (Dr. rer. nat.) from the University of Karlsruhe. His main research interests are databases in distributed, heterogeneous information systems, both from a technical and from an application's point of view.

Tom Quellenberg works as a research assistant in the database research group at FZI, Karlsruhe. He holds a Diploma degree (Dipl. Inform.) from the University of Bremen and is mainly interested in workflow management and object oriented database systems. 